\title{
Evaluación de la incidencia de brucelosis en Ganado ovino, caprino, y bovino en México (2017-2019)
}

\section{Evaluation of Brucellosis incidence in sheep, goat, and bovine cattle in Mexico (2017-2019)}

\author{
Isi I. Ordóñez-Velázquez ${ }^{a}$, Sylvia Martínez-Hernández ${ }^{a, b}$, Ana P. Martínez-Falcón ${ }^{a}$
}

\begin{abstract}
:
Brucellosis is an infectious disease caused by bacteria of the genus Brucella which have negative effects on humans, domestic and wildlife animals. The objective is to analyze the current status of Brucella in Mexico, through the analysis of official government databases in order to describe the states with the highest incidence of Brucellosis during the period 2017-2019. The data were obtained from government websites were analyzed implementing a generalized linear analysis with quasipoisson distribution using the statistical program R (R core team). In Mexico, the states with the highest incidence of brucellosis were Sinaloa with $35.70 \%$ for sheep and $55.11 \%$ for goats, while Jalisco presented the highest percentage of $60.85 \%$ for cattle. Nationally, these two states are leading the incidence of brucellosis. The statistical analysis revealed that there are no significant differences in the number of quarantines presented, while the analysis by type of cattle revealed that cattle have the highest incidence of brucellosis compared to sheep and goats.
\end{abstract}

Keywords:

Brucellosis, cattle, ecology, México

\section{Resumen:}

La Brucelosis es una enfermedad infectocontagiosa que se da por bacterias del género Brucella, las cuales tienen afectaciones negativas en el hombre, animales domésticos y fauna silvestre. El objetivo es analizar el estatus actual de Brucella en México, mediante el análisis de bases de datos oficiales gubernamentales, con la finalidad de describir los estados con mayor incidencia de Brucelosis durante el período 2017- 2019. Los datos se obtuvieron de páginas gubernamentales y el análisis estadístico se empleó el programa $\mathrm{R}(\mathrm{R})$, mediante un análisis lineal generalizado con distribución quasipoisson. En México los estados con mayor incidencia de brucelosis fueron; Sinaloa con $35.70 \%$ para ganado ovino y un $55.11 \%$ para ganado caprino, mientras que Jalisco $60.85 \%$ para ganado bovino. Estos estados lideran la cantidad de cuarentenas probables y confirmadas en todo el país. El análisis estadístico reveló que no existen diferencias significativas en el número de cuarentenas presentados, mientras que el análisis por tipo de ganado reveló que el ganado bovino tiene mayor incidencia de brucelosis respecto al ganado ovino y caprino.

\section{Palabras Clave:}

Brucelosis, ganado, ecología, México

\section{Introducción}

La brucelosis es una enfermedad infectocontagiosa originada por bacterias del género Brucella las cuales tienen afectaciones en humanos, animales domésticos y fauna silvestre especialmente mamíferos pequeños, dentro de la familia Brucellaceae existen 6 especies descritas (B. melitensis, B. abortus, B. suis, B. ovis, $B$. canis y B. neotomae), cada una de estas especies generalmente tienen un hospedero específico 1, 2 .

Sin embargo se han encontrado casos en los que existe un contagio cruzado entre diferentes especies de hospedero, un claro ejemplo de esto es $B$. melitensis que infecta principalmente a caprinos y por infección cruzada, vacas y cerdos, $B$. abortus por lo general infecta ganado vacuno, pero puede infectar cabras, y $B$. suis infecta cerdos, estas cepas llegan a infectar

\footnotetext{
a Universidad Autónoma del Estado de Hidalgo, Instituto de Ciencias Básicas e Ingeniería. Ciudad del Conocimiento, Carretera PachucaTulancingo, km 4.5. Col. Carboneras, Mineral de la Reforma, Hidalgo. México. Isi Iris Ordóñez Velázquez, https://orcid.org/0000-00018428-6437, or286728@uaeh.edu.mx; Sylvia Martínez Hernández, sylvia_martinez10436@uaeh.edu.mx, https://orcid.org/0000-0002-33134318; Ana Paola Martínez Falcón, https://orcid.org/0000-0003-3307-3989, ana_martinez6052@uaeh.edu.mx
} 
animales sanos o a rebaños cercanos; su modo de trasmisión es vía oral, contacto directo con fluidos o restos de aborto infectados, ingerir productos lácteos crudos contaminados y entrada por vía aérea debido a la contaminación ambiental 3, 4. Estas especies tienen un tiempo de incubación de dos semanas a meses, debido a que cuentan con la capacidad de evadir al sistema inmune, generando grandes afectaciones que van desde fiebres altas y ondulantes, debilidad corporal, abortos, descendencia débil, infertilidad, orquitis, epididimitis, trastornos osteoarticulares, meningitis, anemia y hasta la muerte si no se trata a tiempo 5,6. Esta zoonosis debe ser controlada debido al alto impacto económico que genera en la industria ganadera, sector salud; así como en campañas de vacunación, cursos, medicamentos para la industria pecuaria, pérdidas monetarias de animales infectados y en tratamientos de pacientes con brucelosis 7. De acuerdo con lo anterior el presente trabajo pretende analizar el estatus actual de brucelosis en México, mediante el análisis de bases de datos oficiales gubernamentales, con la finalidad de describir los estados con mayor incidencia durante el período 2017- 2019.

\section{Métodos}

Se analizaron bases de datos que contenían el número de cuarentenas probables y confirmadas de Brucelosis en ganado ovino, bovino y caprino, reportados en los últimos tres años en México (2017-2019). Las bases de datos fueron obtenidas de Informes Trimestrales reportados por los Coordinadores Estatales de la Dirección de Campañas Zoosanitarias (DCZ), Secretaría de Agricultura y Desarrollo Rural (SADER) y El Servicio Nacional de Sanidad, Inocuidad y Calidad Agroalimentaria (SENASICA), solicitadas previamente mediante comunicaciones oficiales; así como una recopilación de datos de libre acceso en las páginas del Gobierno de México (https://www.gob.mx/senasica). Para el análisis estadístico se usó el programa $R$ software ( $R$ core team, 2020) para mostrar si existen diferencias significativas en la incidencia de brucelosis tanto por año cómo por especie de ganado mediante un análisis lineal generalizado con distribución quasipoisson.

\section{Resultados y discusión}

El análisis estadístico reveló que no existen diferencias significativas en el número de cuarentenas presentadas en cada periodo $\left(X^{2}=1535, g l=2, P=0.95\right)$, ya que no hubo un incremento considerable en la cantidad de cuarentenas probables y confirmadas. Por el contrario, la diferencia es significativa al analizar el tipo de ganado $\left(X^{2}=423227, g l=2, P=0.0001\right)$. Los bovinos presentan un número notablemente más alto en contraste con los ovinos y caprinos (fig.1). Por lo tanto, la incidencia de cuarentenas en el ganado ovino y caprino ha sido estable en los tres años.

Del 2017 al 2018 hubo un descenso de cuarentenas en el ganado ovino, caprino y bovino disminuyendo un 2, 8 y $12 \%$, respectivamente. Mientras que del 2018 al 2019 se presenta nuevamente una disminución de $1 \%$ de cuarentenas en el ganado caprino, por el contrario, los ovinos aumentaron un $8 \%$ y los bovinos un $5 \%$ (fig. 1 ).

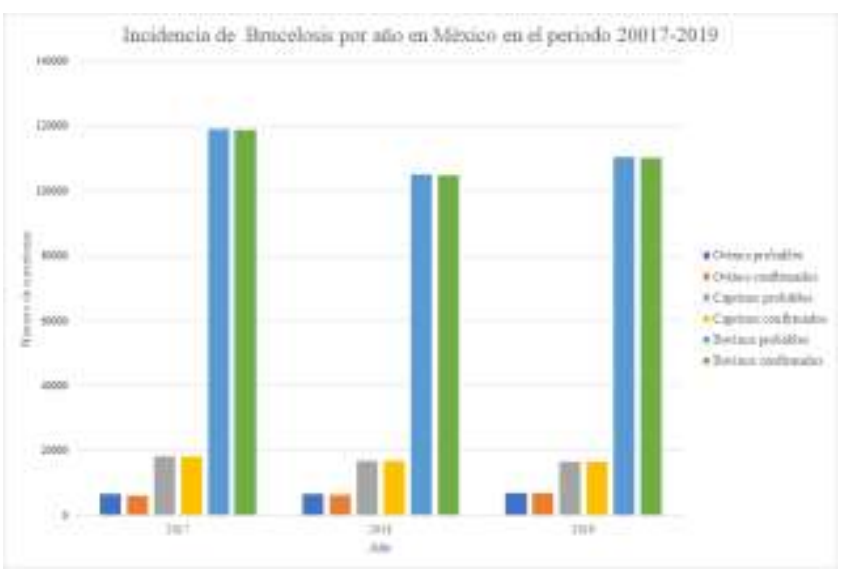

Figura 1. Total de cuarentenas aisladas (probables) y cuarentenas erradicadas (confirmadas) en los años 2017, 2018, 2019.

De acuerdo al análisis de datos, se establecieron los estados de mayor incidencia por cada tipo de ganado. En el ganado ovino los estados con mayor incidencia son Sinaloa con $35.70 \%$ y Jalisco con $27,22 \%$, y con menor incidencia Chiapas, Sonora y Baja California con $0.24,0.15$ y $0.02 \%$, respectivamente (fig. 2 ).

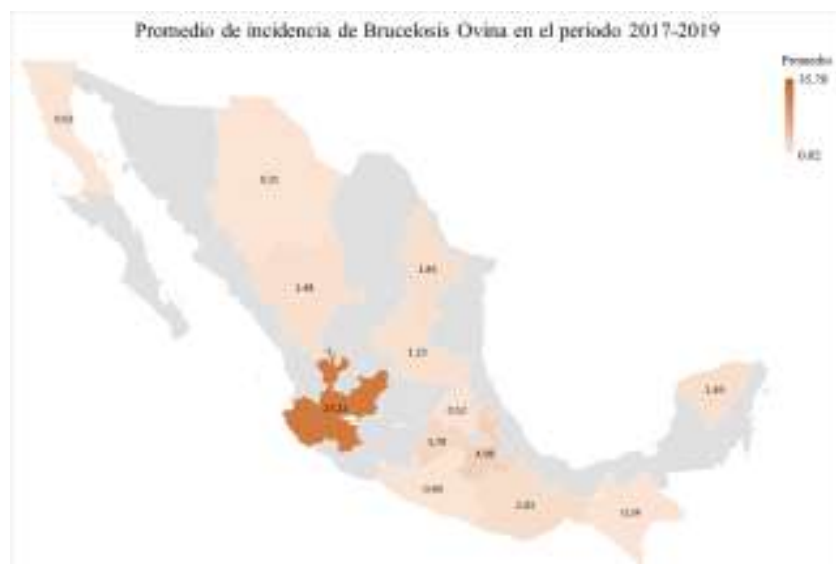

Figura 2. Mapa del promedio de casos totales de brucelosis ovina del periodo 2017-2019.

Para el ganado caprino los estados con mayor incidencia son Sinaloa con el $55.11 \%$, seguido de Nuevo 
León con $11.17 \%$ y San Luis Potosí con $10.46 \%$, y con menor incidencia están Chihuahua e Hidalgo con 0.12 y $0.08 \%$, respectivamente.

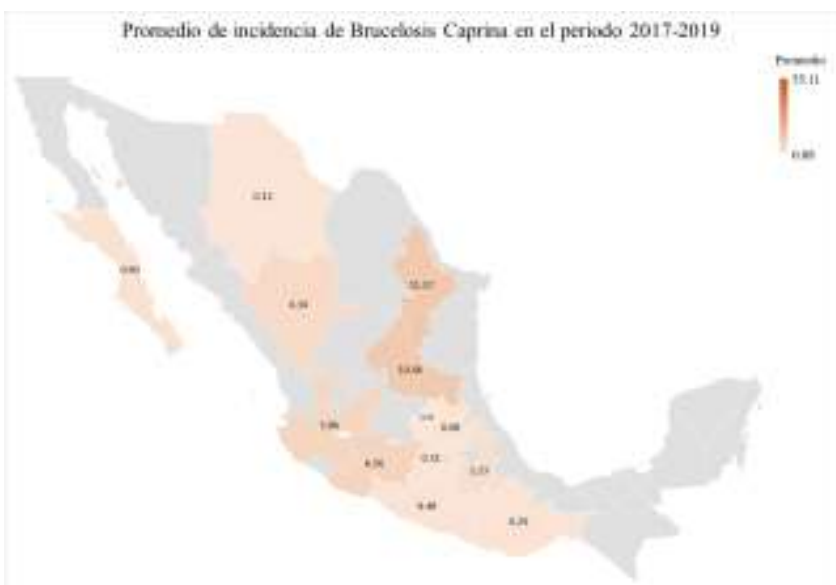

Figura 3. Mapa de casos promedio de brucelosis caprina del periodo 2017-2019.

Finalmente, los estados con mayor incidencia de brucelosis en ganado bovino fueron Jalisco con $60.85 \%$, Nayarit con $6.74 \%$, mientras que Hidalgo presentó la menor incidencia con $0.05 \%$. (fig. 4). Jalisco se encuentra en estado de alarma, ya que mostró un aumento en la incidencia de cuarentenas durante el periodo analizado.

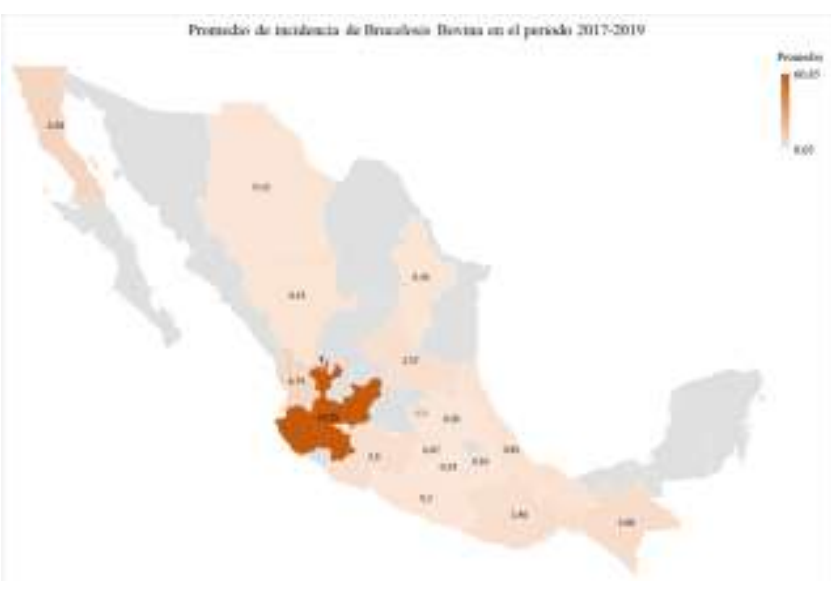

Figura 4. Mapa de casos promedio de brucelosis bovina del periodo 2017-2019.

El género Brucella se encuentra distribuido en todo el mundo, aunque en algunos países se encuentra erradicado, lo cual no es el caso de México 8. Según las bases de datos analizadas, existen estados sin presencia de brucelosis en los tres años analizados (Aguascalientes, Coahuila, Colima, Guanajuato, Tlaxcala y Tamaulipas), mientras que el $28.88 \%$ del territorio nacional se reconoce en fase de erradicación
(Campeche, Guerrero, Quintana Roo y Yucatán, algunas zonas de Baja California, Chiapas, Hidalgo, Estado de México, Puebla, Oaxaca y Querétaro) 9.

Los estados con mayor incidencia de casos probables y confirmados son Jalisco y Sinaloa. Coincidentemente, estos estados son los mayores productores de ganado, por lo que representan una alta probabilidad de generar brotes. Algunos estudios demuestran que la alta prevalencia de la enfermedad se debe a la densidad o tamaño de los rebaños como un factor de alto riesgo 10 12.

La brucelosis en ganado se debe a factores ambientales, sanitarios, y muchas veces a la falta de información de los productores. Un estudio demostró que el $93 \%$ del personal y dueños que trabajan en los hatos no presentaban un conocimiento sobre la enfermedad 13.

La erradicación total se complica, ya que las especies $B$. melitis, B. abortus y $B$. ovis, se encuentran en el ambiente de manera natural y son las especies que afectan principalmente al ganado bovino, caprino y ovino. Además, presentan un potencial zoonótico alto a moderado, y un extensivo periodo de supervivencia en el ambiente de hasta de 2 meses en tierra húmeda 4, 6 , 14.

Existen manuales de buenas prácticas de ganadería que se encuentran de manera gratuita en plataformas gubernamentales oficiales, sin embargo, aunque estas actividades se realicen de manera adecuada, la alta cantidad de animales que se maneja aumenta la probabilidad de surgimientos de brotes y permanencia de enfermedades zoonóticas 15-17.

Es importante resaltar que la ganadería extensiva o intensiva que se practica en nuestro país no siempre se realiza bajo las mejores condiciones sanitarias, por lo que la presencia de brucelosis en un hato puede diseminarse relativamente rápido (mediante vectores como los roedores), al ganado de granjas cercanas. Los roedores son expuestos a cepas del género Brucella al estar en contacto con secreciones genitales o mamarias de ganado enfermo, además de que Brucella tiene la capacidad de entrar por las conjuntivas o heridas de la piel 18.

Por otro lado, los roedores pueden portar diferentes especies de Brucella sp., lo cual hace difícil controlar la zoonosis de estas enfermedades, debido a sus hábitos de dispersión y de poblaciones con alta densidad de individuos 19.

Las especies del género Brucella por sí mismas pueden permanecer en diferentes ambientes y en períodos largos, como el suelo y el estiércol (80 días), depósitos de lana (110 días) y fetos abortados que quedan en la sombra (6-8 meses) 4 . 
La brucelosis juega un factor importante en el impacto de la ganadería ya que de acuerdo con SENASICA, en México cada año aumenta la producción de carne para consumo y exportación, incrementando la probabilidad de generar brotes de la enfermedad con afectación al ganado, a las personas que trabajan en estos sectores, y por último a las personas que consumen frescos y derivados ganaderos provenientes de animales infectados 5, 18, 19.

El impacto adverso en el sector económico y en el sector salud es alto, tan sólo en el 2000 se reportaron pérdidas en América Latina por 600 millones de dólares sólo por ganado caprino (por mortalidad de la progenie, pérdidas de esterilidad y por pérdidas en la disminución de la producción lechera) 20.

En México no hay cifras oficiales disponibles de las pérdidas económicas a nivel nacional o regional. Sin embargo, una investigación realizada en unidades de producción lechera con diferentes subprogramas gubernamentales para el control de la enfermedad estima el coste financiero entre 33.58 y 70 USD/animal/año, sólo por los eventos de falla reproductiva 21 .

\section{Conclusiones}

El $71.12 \%$ del territorio nacional presenta brucelosis activa pese a las campañas de vacunación y a la difusión de información. Los esfuerzos que se realizan para erradicar la enfermedad no son suficientes; a lo anterior se le suma la desinformación de los ganaderos para prevenir y controlar la brucelosis, y que no siempre se realizan pruebas para diagnosticar la enfermedad. Lo que resulta en pérdidas económicas importantes en el sector y un potencial riesgo a la salud humana. Por lo que se recomienda prestar atención en los estados de alarma antes mencionados; realizando diagnósticos tempranos y oportunos, para esto es fundamental tener personal capacitado e instalaciones adecuadas, además de tomar precauciones en el monitoreo de las importaciones y movimiento de ganado. Es muy importante fortalecer los programas y apoyos estatales para minimizar o subsanar las pérdidas económicas, lo que incentivaría a los ganaderos a declarar los posibles casos de brucelosis.

\section{Referencias}

[1] Garrity G. (2001). Family III. Brucellaceae Breed, Murray and Smith 1957. En M. J. Corbel \& M. Bana (Eds.), Bergey's Manual of Systematic Bacteriology. 2001; vol. 2, pp. 370-381. Michigan, USA: Springer.

[2] Godfroid J, DeBolle X, Roop R M, O’Callaghan D, Tsolis R M, Baldwin $\mathrm{C}$, et al. The quest for a true one health perspective of brucellosis. Rev. Sci. Tech. 2014; 33(2): 521-38.
[3] Álvarez J, Sáez J L, García N, Serrat C, Pérez Sancho M, González S, et al. Management of an outbreak of brucellosis due to B. melitensis in dairy cattle in Spain. Res Vet Sci. 2011; 90: 208-11

[4] Álvarez-Hernández N E, Díaz-Flores M, Ortiz-Reynoso M. Brucelosis, una zoonosis frecuente. Rev. Med. Inv. 2015; 3(2): 129-133. https://doi.org/10.1016/j.mei.2015.07

[5] Guzmán Hernández R L, Contreras Rodríguez A, Ávila Calderón E D, Morales García M R et al. Brucelosis: zoonosis de importancia en México. Rev. Chilena Infectol. 2016; 3(6): 656-662. https://scielo.conicyt.cl/pdf/rci/v33n6/art07.pdf

[6] Byndloss M X, Tsolis R M. Brucella spp. Virulence Factors and Immunity. Annu. Rev. Anim. Biosci. 2016; 4(1): 111-127. https://doi.org/10.1146/annurev-animal-021815-111326

[7] Laplume H, Sardi F, Jacob N, Garro S, Lucero N, Reynes E, Amartino L. Enfermedades infecciosas brucelosis GUIA PARA EL EQUIPO DE SALUD. 2013. http://www.msal.gob.ar/images/stories/bes/graficos/0000000304cntguia-medica-brucelosis.pdf

[8] Rodríguez Zapata M, Solera Santos J, Sánchez Martínez L, Solís García del Pozo J, Álvarez-Mon Soto M. Brucelosis. Medicine Programa de Formación Médica Continuada Acreditado, 2002; 8(65), 3455-3463. doi:10.1016/s0304-5412(02)70644-6

[9] SENASICA. Servicio de Sanidad, Inocuidad y Calidad (2020). Situación actual del control de la brucelosis en México. https://www.gob.mx/senasica/documentos/situacion-actual-delcontrol-de-la-brucelosis-en-mexico

[10] SAGARPA. Secretaría de Agricultura y Desarrollo Rural (2019). Producción ganadera, Datos abiertos, Estadística de Producción Ganadera. http://infosiap.siap.gob.mx/gobmx/datosAbiertos_p.php

[11] Poulsen K P, McNulty C M, Trueba G, Barragán V, Zabala C, Hutchins F T, López L. Short Report: Brucellosis in Dairy Cattle and Goats in Northern Ecuador. Am. J. Trop. Med. Hyg. 2014; 90(4): 712 715. https://doi.org/10.4269/ajtmh.13-0362

[12] Ayala E, Tobar L. Incidencia de brucelosis bovina (Brucella abortus) en los hatos lecheros de la Asociación Rancheros del Norte, Parroquia El Carmelo, Cantón Tulcán, Provincia del Carchi. Tesis. Universidad Politécnica Estatal de Carchi. 2011. 112 p.

[13] Zambrano Aguayo M D, Pérez Ruano M, Rodríguez Villafuerte X. Brucelosis Bovina en la Provincia Manabí, Ecuador. Estudio de los Factores de Riesgo. Rev. Inv. Vet. Perú. 2016; 27(3): 607-617. https://doi.org/10.15381/rivep.v27i3.11995

[14] Serra J, Pujol R, Godoy P. Estudio cero epidemiológico de la brucelosis en un área rural endémica. Enferm. Infecc. Microbiol. Clin. 2000; 18(2): 74-78

[15] SENASICA, SAGARPA. Servicio de Sanidad, Inocuidad y Calidad, Secretaría de Agricultura y Desarrollo Rural. (2014). Manual de Buenas Prácticas Pecuarias, Sistema de Explotación Extensivo y SemiExtensivo de Ganado Bovino de Doble Propósito. http://publico.senasica.gob.mx/?doc $=21454$

[16] Hoth J. (2012) Buenas prácticas ganaderas en México / Ganadería beneficiosa Prácticas de gestión en México. Comisión para la Cooperación Ambiental de América del Norte (CCA). Montreal. https://www.researchgate.net/publication/235920888 Buenas practica s_ganaderas_en_Mexico_Beneficial_Livestock_Management_Practice $\underline{\text { s in Mexico\#fullTextFileContent }}$

[17] Lagoma Lorén L. (2009). Zoonosis laborales: riesgos de exposición a agentes biológicos en ganadería. Seguridad y salud en el Trabajo. 2009; 55: 42-47.

[18] SAGARPA, Secretaría de Agricultura y Desarrollo Rural (2018). Reporte del mercado de Carne de bovino. Centro de información de mercados agroalimentarios. https://www.cima.aserca.gob.mx/work/models/cima/pdf/cadena/2018/ Reporte mercado bovino 200618.pdf 
[19] Días Aparicio E. Epidemiología de la brucelosis causada por Brucella melitensis, Brucella suis y Brucella abortus en animales domésticos. Rev. Sci. Tech. https://pdfs.semanticscholar.org/7912/0de6d16d5ff33ed19b6051f3204 ea6373d9d.pdf

[20] Sreevatsan S, J B Bookout, F Ringpis, V S Perumaalla, T A Ficht, L $\mathrm{G}$ Adams. A multiplex approach to molecular detection of Brucella abortus and/or Mycobacterium bovis infection in cattle. J. Clin. Microbiol. $\quad 2000 ; \quad 38(7): \quad 2602-2610$. https://jcm.asm.org/content/jcm/38/7/2602.full.pdf

[21] Xolalpa Campos V M, Pérez Ruano M, Córdova Izquierdo A Evaluación de las pérdidas económicas por eventos de falla reproductiva asociadas a brucelosis bovina en hembras y explotaciones de la cuenca lechera de Tizayuca, Hidalgo, México. Rev. Cientif. FCV-LUZ. $\quad 2010 ; \quad 20(2)$ : 20190 . https://www.redalyc.org/articulo.oa?id=95912322012 